\title{
The Effects of Doppler Shift on DOA Estimation in TDRSS and its Compensation Method
}

\author{
Xiaoheng Tan ${ }^{1}$, Qian $\mathrm{Qu}^{2}$, Dong Jiang ${ }^{3}, \mathrm{Li} \mathrm{Wen}^{3}$ and Lu Deng ${ }^{4}$ \\ College of communication engineering, Chongqing University, Chongqing, \\ 400044, China \\ 907843941@qq.com
}

\begin{abstract}
In the reverse link of Tracking and Data Relay Satellite System (TDRSS), Doppler effects in Frequency-Division Multiplexing (FDM) signal cause different frequency shift in different channels. In this work, it analyzes the influence of Doppler effects among FDM channels and establishes a mathematical model of Direction of Arrivals (DOA) estimation error while existing different frequency shift among channels. The results show that different frequency shift impacted on the correctness of DOA estimation: the direction of signals and MUSIC spectrum change periodically and non-uniform frequency shift can ruduce spectral peak. This paper proposes an improved PLL based on Extend Kalman Filter (EKF) to compensate Doppler shift between channels. The final sumulation prove that the improved PLL can correct the non-uniform frequency shift.
\end{abstract}

Keywords: TDRSS, DOA, Doppler Shift, MUSIC, EKF

\section{Introduction}

The reverse link of Tracking and Data Relay Satellite System (TDRSS) is used to transmit data from user satellites to ground stations. Usually, Tracking and Data Relay Satellite (TDRS) use phased array antennas to track and forward multitargets. In order to reduce the payload and processing work of satellite, ground Digital Beam-Forming (DBF) is used frequently [1-2]. In this system, there are 30 independent array elements on TDRS and the datas of 30 array elements are sent to ground station through FDM scheme. Then DOA estimation and DBF can be realized to enhance the SNR of received signals at the ground station.

Doppler effect is common in wireless communication especially satellite communication system[3]. Although TDRS is on geostationary orbit, the existing small inclination can cause relative motion between the satellite and earth. As the communication frequency is very high (Ka band is used in the reverse link of TDRSS), the frequency shift of carrier can't be ignored. For FDM scheme, there are 30 carriers, and every channel gets different frequency offset. The DOA estimation accuracy can be influenced badly due to the additional frequency offset in every channel, it can become worse as time passes.

DOA estimation accuracy is influenced by the number of the snapshots, array elements, and the SNR values etc. MUSIC algorithm is classical and performances well [6]. For subspace-based algorithms, array sensors' position error may severely influence the estimation result [7]. To reduce the influence and promote the performance, there are many enhanced DOA estimation algorithms or methods [8] [9] [10]. But in the background of TDRSS, there are few papers considering the influence on DOA estimation as the using of FDM scheme. In [11], multi-channel group delay distortion is put forward,

Received (March 30, 2017), Review Result (November 24, 2017), Accepted (November 28, 2017) 
which has an impact on the performance of TDRSS. AM/PM conversion is also a error source to DOA estimation in TDRSS [12].

This study is motivated by the fact that previous studies [11] [12] didn't consider the Doppler effects in the reverse link of TDRSS. This paper is organized as follows. In Section 2, TDRSS model with Doppler Shift is established, and it makes a detailed introduction of mathematical derivation of MUSIC algorithm with different Doppler Shift in every channel. Section 3 proposes the compensation method with the structure of improved carrier tracking loop based on Kalman Filter.

Sections 4 simulates the reverse link of TDRSS and analyzes the influence to DOA estimation with different Doppler Shift and the effect of compensation. Section 5, as the conclusion part, analyzes the results of the simulation. It shows that absolute Doppler Shift and relative Doppler Shift can bring the same effects to DOA estimation, and our compensation method is effective to improve the performance of DOA.

\section{The Effects of Doppler Shift on DOA}

\subsection{Analysis of the TDRSS Model}

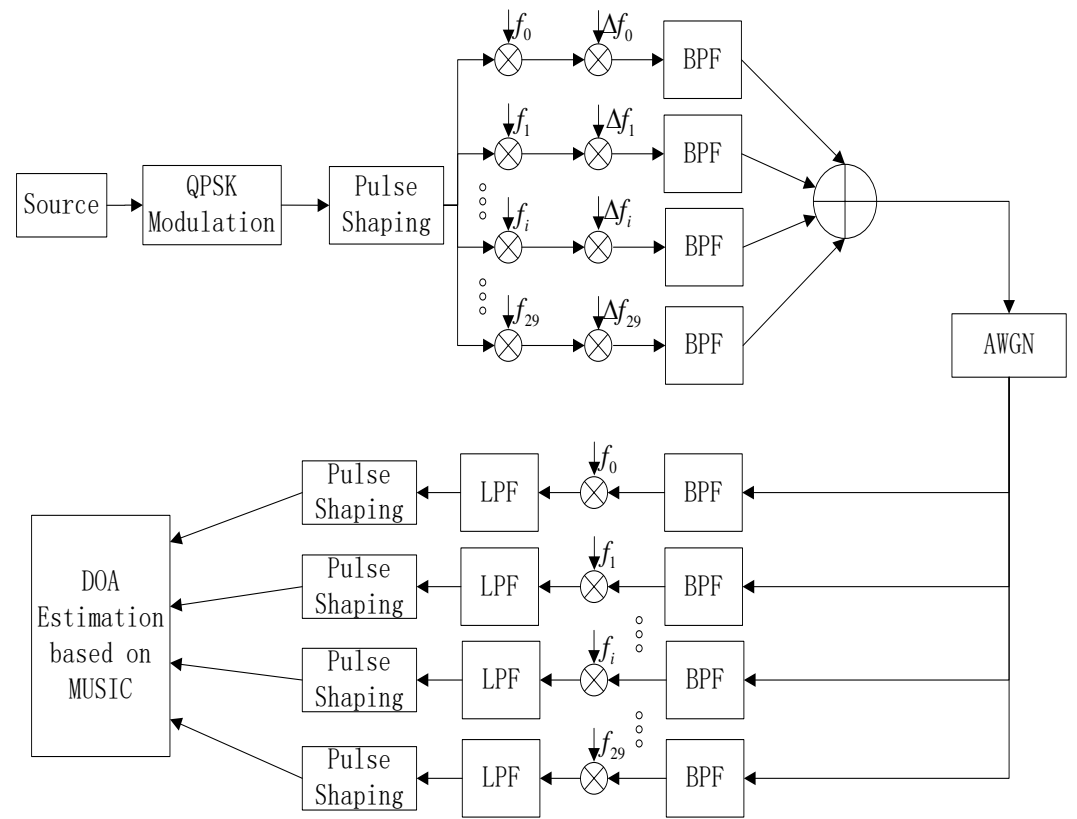

Figure 1. The System Model of Return Link in TDRSS

In order to describe the reverse link of TDRSS, we consider the model as Figure 1 shows.

We represent the input signal as $s(t)$, and the signal which the array receives can be showed as:

$$
s_{i}(t)=s(t) e^{-j(i-1) \Delta \varphi}
$$

Assuming the Doppler Shift on channel $i$ is $\Delta f_{i}$, and the signal which is transmitted through the Satellite-Earth link can be described as:

$$
x_{i}(t)=s(t) e^{-j(i-1) \Delta \varphi} e^{j 2 \pi \Delta f_{i} t}
$$

So the 30 array elements signal is: 


$$
\begin{aligned}
& \mathbf{X}(t)=\left[x_{0}(t), x_{1}(t), \mathrm{L}, x_{29}(t)\right] \\
& =s(t)\left[e^{j 2 \pi \Delta f_{0} t}, e^{j 2 \pi \Delta f_{1} t} e^{-j(2-1) \Delta \varphi}, \mathrm{L}, e^{j 2 \pi \Delta f_{29} t} e^{-j(30-1) \Delta \varphi}\right]
\end{aligned}
$$

The abbreviation of formula (3) is:

$$
\mathbf{X}(t)=\mathbf{D}(t) \mathbf{A}(\theta) s(t)
$$

And:

$$
\mathbf{D}(t)=\operatorname{diag}\left[e^{j 2 \pi f d_{0} t}, e^{j 2 \pi f d_{1} t}, \mathrm{~L}, e^{j 2 \pi f d_{29} t}\right]
$$

$f d_{0}, f d_{1} \ldots f d_{29}$ are the Doppler Shifts in every channel. Matrix $\mathbf{D}(t)$ reflects the phase rotation caused by Doppler Shift during the transmission. $\mathbf{A}(\theta)$ is the direction matrix. Considering the effect of Gauss white noise, formula (4) can be rewritten as:

$$
\mathbf{X}(t)=\mathbf{D}(t) \mathbf{A}(\theta) s(t)+\mathbf{n}(t)
$$

After the remote transmission by FDM method, $\mathbf{D}(t)$ exists in formula (6). Doppler Shift can bring phase error to output signals, which can also make the performance of DOA worse.

\subsection{Analysis of DOA Estimation based on MUSIC}

In formula (6), if we ignore the Doppler Shift in every channel, then $\mathbf{D}(t)=\mathbf{I}$. Considering the influence of Doppler Shift, $\mathbf{A}(\theta)$ changes to $\mathbf{D}(t) \mathbf{A}(\theta)$. So in the spectrum function of MUSIC algorithm, if we use $\mathbf{D}(t) \mathbf{A}(\theta)$ instead, there must be error direction. Actually, the covariance matrix is:

$$
\hat{\mathbf{R}}_{\mathrm{Xx}}=\mathbf{D}(\mathbf{t}) \mathbf{R}_{\mathrm{Xx}} \mathbf{D}^{\mathrm{H}}(\mathbf{t})
$$

And :

$$
\begin{aligned}
& \mathbf{D}(t)=\operatorname{diag}\left[e^{j 2 \pi f d_{0} t}, e^{j 2 \pi f d_{1} t}, \mathrm{~L}, e^{j 2 \pi f d_{29} t}\right] \\
& =e^{j 2 \pi f d_{0} t} \operatorname{diag}\left[e^{j 0}, e^{j 2 \pi\left(f d_{1}-f d_{0}\right) t}, \mathrm{~L}, e^{j 2 \pi\left(f d_{30}-f d_{0}\right) t}\right] \\
& =e^{j 2 \pi f d_{0} t} \operatorname{diag}\left[e^{j 0}, e^{j 2 \pi \Delta f_{1} t}, \mathrm{~L}, e^{j 2 \pi \Delta f_{29} t}\right] \\
& =e^{j 2 \pi f d_{0} t} \mathbf{D}^{\prime}
\end{aligned}
$$

$\Delta f_{1} 、 \Delta f_{2} \ldots \Delta f_{29}$ are the relative Doppler Shifts between reference channel and other channels.

So:

$$
\hat{\mathbf{R}}_{\mathrm{Xx}}=\mathbf{D}(\mathbf{t}) \mathbf{R}_{\mathrm{XX}} \mathbf{D}^{\mathrm{H}}(\mathbf{t})=\mathbf{D}^{\prime}(\mathbf{t}) \mathbf{R}_{\mathrm{XX}} \mathbf{D}^{\prime \mathbf{H}}(\mathbf{t})
$$

Therefore, only the relative Doppler Shift can have an effect on the performance of DOA estimation. 


$$
\mathbf{R}_{\mathbf{x x}}=E\left[\mathbf{X}(\mathbf{t}) \mathbf{X}^{\mathbf{H}}(\mathbf{t})\right]=\mathbf{A}(\boldsymbol{\theta}) \mathbf{R}_{\mathrm{ss}} \mathbf{A}^{\mathbf{H}}(\boldsymbol{\theta})+\sigma^{2} \mathbf{I}
$$

$\mathbf{R}_{\mathbf{X X}}$ is the covariance matrix when there is no Doppler Shift, and $\mathbf{R}_{\mathrm{ss}}=E\left[s(t) s^{H}(t)\right]$ is the correlation matrix of the source.

In order to facilitate the analysis, we consider the DOA estimation with one source. Eigenvalue $\left\{\lambda_{0}, \lambda_{1}, \mathrm{~L}, \lambda_{29}\right\}$ can be calculated with eigen-decomposition of $\hat{\mathbf{R}}_{\mathbf{x x}}$. And it's corresponding eigenvector is $\left\{e_{0}, e_{1}, \mathrm{~L}, e_{29}\right\}$.

Thus:

$$
\mathbf{A}^{\mathbf{H}}(\boldsymbol{\theta}) \mathbf{D}^{\mathbf{H}}(\boldsymbol{\theta}) e_{j}=0 \quad j=1,2, \mathrm{~L} 29
$$

The actual spectrum of MUSIC is:

$$
P=\frac{1}{\mathbf{A}^{\mathbf{H}}(\boldsymbol{\theta}) \mathbf{E}_{\mathbf{N}} \mathbf{A}^{\mathbf{H}}(\boldsymbol{\theta})}
$$

$\mathbf{E}_{\mathbf{N}}$ is the orthogonal projection matrix of noise space:

$$
\mathbf{E}_{\mathbf{N}}=\sum_{j=1}^{29} e_{j} e_{j}^{H}=\mathbf{E}-\frac{\mathbf{D}(\mathbf{t}) \mathbf{A}\left(\boldsymbol{\theta}_{\mathbf{0}}\right) \mathbf{A}^{\mathbf{H}}\left(\boldsymbol{\theta}_{\mathbf{0}}\right) \mathbf{D}^{\mathbf{H}}(\mathbf{t})}{\sqrt{30}}
$$

So:

$$
\mathbf{A}^{\mathbf{H}}(\boldsymbol{\theta}) \mathbf{E}_{\mathbf{N}} \mathbf{A}(\boldsymbol{\theta})=N-\frac{\left|\mathbf{A}(\boldsymbol{\theta}) \mathbf{D}(\mathbf{t}) \mathbf{A}^{\mathbf{H}}\left(\boldsymbol{\theta}_{\mathbf{0}}\right)\right|^{2}}{\sqrt{30}}
$$

Besides:

$$
\left|\mathbf{A}(\boldsymbol{\theta}) \mathbf{D}(\mathbf{t}) \mathbf{A}^{\mathbf{H}}\left(\boldsymbol{\theta}_{\mathbf{0}}\right)\right|=\left|\sum_{i=0}^{29} \exp \left(j 2 \pi \Delta f_{i} t\right) \exp \left[j(2 \pi d / \lambda)\left(\sin \theta_{0}-\sin \theta\right)\right]\right|
$$

$\theta_{0}$ is the real direction of source, and $\theta$ is the direction estimated by MUSIC. In formula (15), usually at the effect of Doppler Shift $\Delta f_{i}$, the estimation direction is not the real direction. That is to say the disagree of Doppler Shift between channels can lead to the error of DOA.

\section{Compensation Method of different Doppler Shift}

In the reverse link of TDRSS, FDM method which brings 30 array elements signals back to earth can also produce additional Doppler Shift. And according to the above mentioned analysis, the absolute Doppler Shift and relative Doppler Shift can bring the same results to DOA estimation.

From (6), the receive signal can be written as:

$$
y_{i}(t)=s(t) e^{-j(i-1) \Delta \varphi} e^{j 2 \pi \Delta f_{i} t}+n_{i}(t)
$$

The SNR of receive signal, and the Doppler Shift in every channel are small (much less than spreading code rate). Since PLL (phase locked loop) is used in carrier tracking frequently, and its theories and technologies are mature, we take PLL as the basic structure in our compensation method. Different from traditional carrier tracking loop, we don't have local carrier, but we regard the receive signals as "local carrier", which can be 
used to track the signal of reference channel. As Extended Kalman Filter can realize nonlinear filtering and have good performance in low SNR environment, we use Extended Kalman Filter instead of loop filter in PLL. And MF (mean filter) is added before Kalman Filter to make the observation value more smooth. Figure 2 is the improved PLL.

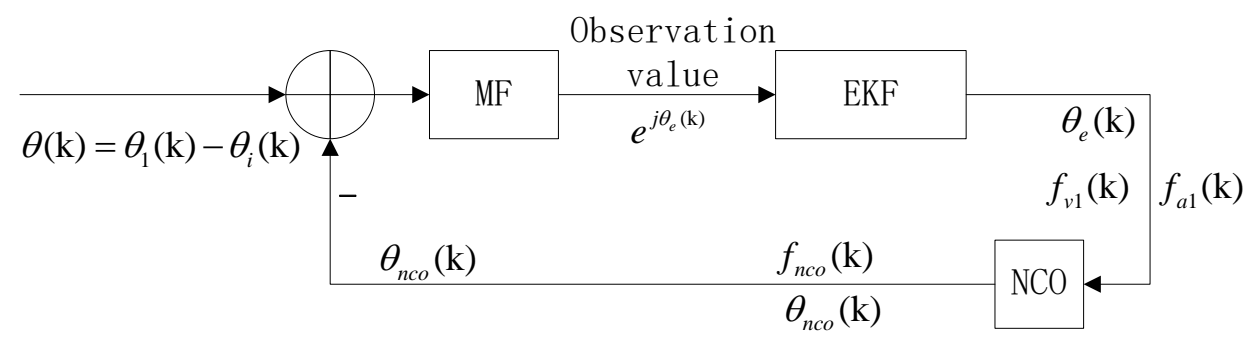

Figure 2. Improved PLL Based on EKF

In our compensation method, reference signal (set as channel 0 ) is mixed with other signals, and $e^{j \theta_{n o}(k)}$ comes from NCO. Phase difference signal is filtered by mean filter and sent to EKF as observation value. So the measurement equation can be:

$$
y_{k}=e^{j \theta_{e}(k)}+v_{k}
$$

In EKF, $x_{k}=\left[\theta_{e}(k), f_{v 1}(k), f_{a 1}(k)\right]^{T}$ is the quantity of state. The relationship among phase, frequency shift and frequency changing rate can be written as:

$$
\begin{aligned}
& \theta(k+1)=\theta(k)+f_{v 1}(k) T_{s}+\frac{f_{a 1}(k) T_{s}^{2}}{2} \\
& f_{v 1}(k+1)=f_{v 1}(k)+f_{a 1}(\mathrm{k}) \mathrm{T}_{s} \\
& f_{a 1}(k+1)=f_{a 1}(k)
\end{aligned}
$$

The update function of NCO can be described as:

$$
\theta_{\text {nсо }}(k+1)=\theta_{\text {nсо }}(k)+\Delta \theta_{\text {nсо }}(k)+f_{\text {nсо }}(k) T_{\text {s }}
$$

From (18) and (21), the phase difference can be:

$$
\begin{aligned}
& \theta_{e}(k+1)=\theta(k+1)-\theta_{n c o}(k+1) \\
& =\theta(k)+f_{v 1}(k) T_{s}+\frac{f_{a 1}(k) T_{s}^{2}}{2}-\left(\theta_{2}(k)+\mathrm{V} \theta_{n c o}(k)+f_{n c o}(k) T_{s}\right) \\
& =\theta_{e}(k)+f_{v 1}(k) T_{s}+\frac{f_{a 1}(k) T_{s}^{2}}{2}-\mathrm{V} \theta_{n c o}(k)-f_{\text {nco }}(k) T_{s}
\end{aligned}
$$

Combine with (19) (20) (21), the equation of state is:

$$
x_{k}=A x_{k-1}+B u_{k-1}+w_{k-1}
$$

The state transition matrix is: 


$$
A=\left[\begin{array}{ccc}
1 & T_{s} & T_{s}^{2} / 2 \\
0 & 1 & T_{s} \\
0 & 0 & 1
\end{array}\right]
$$

Input matrix B is:

$$
B=\left[\begin{array}{cc}
-1 & T_{s} \\
0 & 0 \\
0 & 0
\end{array}\right]
$$

Input signal $u_{k}$ is:

$$
u_{k}=\left[\begin{array}{c}
\mathrm{V} \theta_{n c o} \\
f_{n c o}
\end{array}\right]
$$

$w_{k}$ is the noise vector of state.

As so far, we have the measurement equation and state equation of EKF. Through the procedure of prediction and correction, the state variables can be updated. With the output of NCO, channels with relative Doppler shift can be corrected.

\section{Simulation and Analyze}

\subsection{System Model and Simulation Parameters}

According to the Doppler Shift calculating model in [15] and the TLE (two line element) of TIANLIAN 1-01 [16], we calculate the Doppler Shift between TDRS and five typical Chinese cities. Table 1 lists the geographical information of the five cities.

Table 1. Geographical Coordinates of 5 Cities in China

\begin{tabular}{cccccc}
\hline City & Beijing & Mohe & Sanya & Wulumuqi & Xichang \\
\hline Longitude(deg) & 116.417 & 122.37 & 109.5 & 87.36 & 102.16 \\
Latitude(deg) & 39.9167 & 53.48 & 18.2 & 43.46 & 27.54 \\
Altitude(km) & 0 & 0 & 0 & 0 & 2 \\
\hline
\end{tabular}

To meet the demand of high transmit rate, Ka band is used in the reverse link of TDRSS. Therefore we set carrier frequency as $f_{0}=26 \mathrm{GHz}$. Figure 3 shows the Doppler Shift between ground station and TDRS in 24 hours. From Table 2, the max Doppler Shift between ground station and TDRS can reach $\pm 800 \mathrm{~Hz}$.

To be efficiency, we simulate the performance of DOA estimation with 10 channels.

In accordance with the demand of the reverse link in TDRSS, we set the SNR=10dB, direction of signal is $0^{\circ}$, and spreading code rate is $3 \mathrm{MHz}$. Then the performance of DOA is simulated with 3 situations: no Doppler Shift, absolute Doppler Shift and relative Doppler Shift. Table 3 lists the value of Doppler Shift in 3situations. 


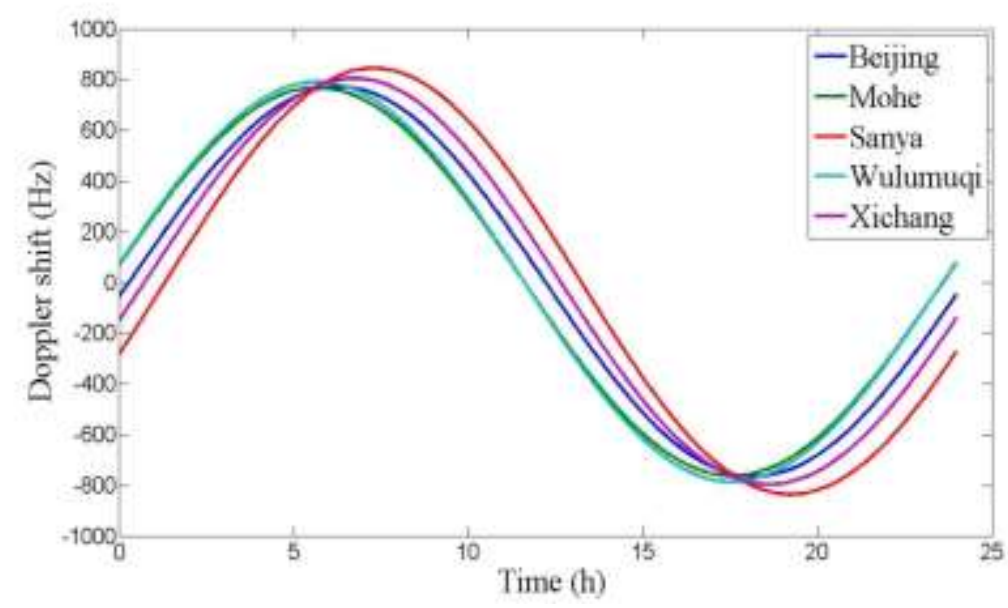

Figure 3. Doppler Shift between TDRS and 5 Cities in China

Table 2. The Maximum Doppler Shift in both Positive and Negative

\begin{tabular}{cccccc}
\hline City & Beijing & Mohe & Sanya & Wulumuqi & Xichang \\
\hline Positive (Hz) & 771.512 & 766.978 & 843.816 & 790.115 & 804.096 \\
Negative (Hz) & 765.925 & 763.154 & 836.713 & 787.305 & 797.077 \\
\hline
\end{tabular}

Table 3. Doppler Shift in 3 Situations

\begin{tabular}{cccc}
\hline Channel & $\begin{array}{c}\text { No Doppler shift } \\
(\mathrm{Hz})\end{array}$ & $\begin{array}{c}\text { Absolute Doppler } \\
\text { shift }(\mathrm{Hz})\end{array}$ & $\begin{array}{c}\text { Relative Doppler } \\
\text { shift (Hz) }\end{array}$ \\
\hline 0 & 0 & 700 & 0 \\
1 & 0 & 710 & 10 \\
2 & 0 & 720 & 20 \\
3 & 0 & 730 & 30 \\
4 & 0 & 740 & 40 \\
5 & 0 & 750 & 50 \\
6 & 0 & 760 & 60 \\
7 & 0 & 770 & 70 \\
8 & 0 & 780 & 80 \\
9 & 0 & 790 & 90 \\
\hline
\end{tabular}

\subsubsection{Simulation Results in 3 Situations}

According to the system model and parameters, MATLAB is adopted to build a simulation model. Figure 4, 5, 6 show the performance of DOA estimation with both 3D and $2 \mathrm{D}$ views. As shown in the three figures, when there is no Doppler Shift, MUSIC can estimate the direction of signals correctly. When absolute Doppler Shift is added to channels, MUSIC fails to estimate the correction direction and the estimation direction changes periodically. Figure 6 shows the same results with Figure 5, therefore relative 
Doppler Shift can bring the same influence as absolute Doppler Shift to estimation performance. It confirms the analysis in part 2.
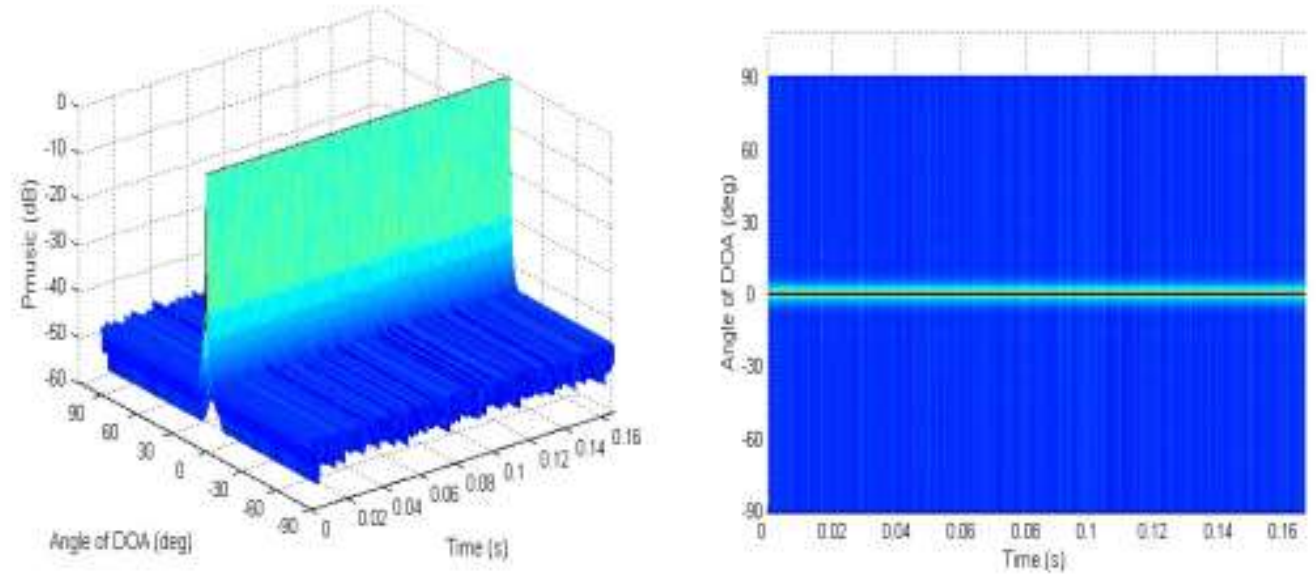

\section{Figure 4. No Doppler Shift}
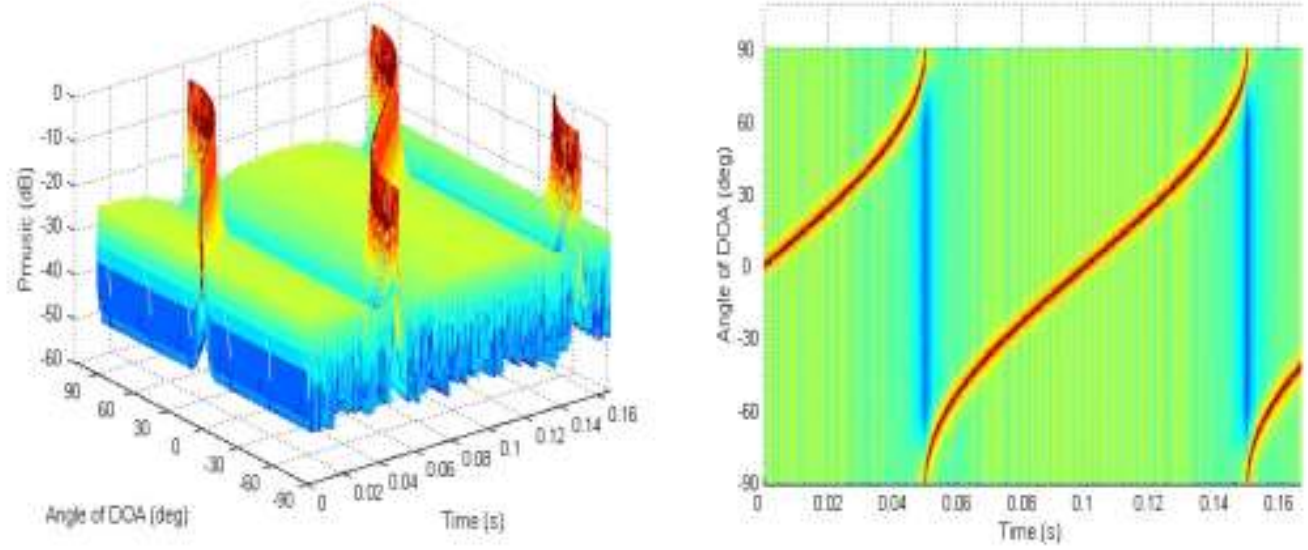

Figure 5. Absolute Doppler Shift
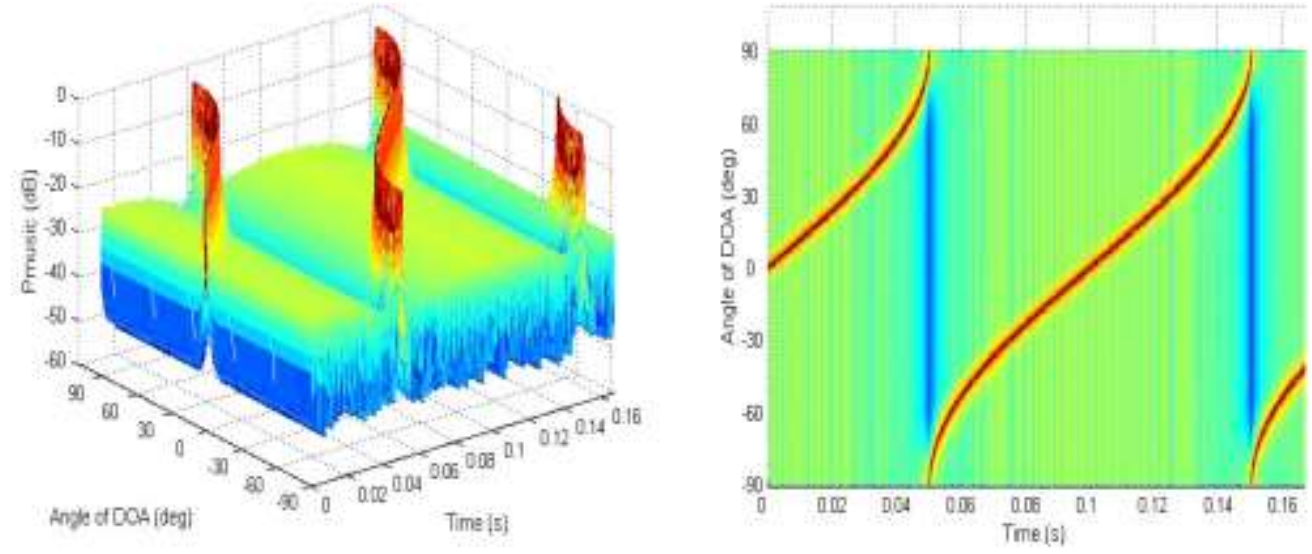

Figure 6. Relative Doppler Shift 


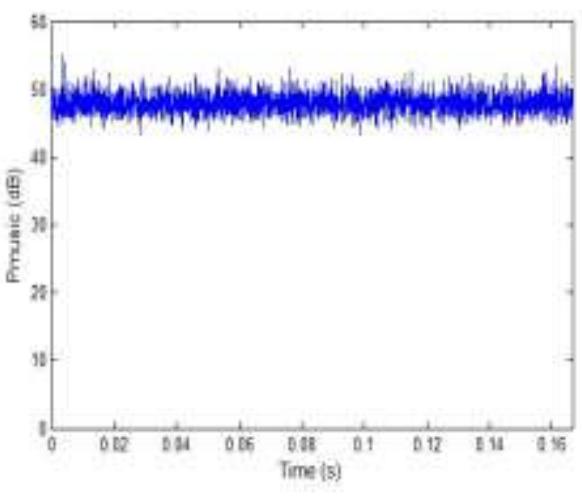

(a)

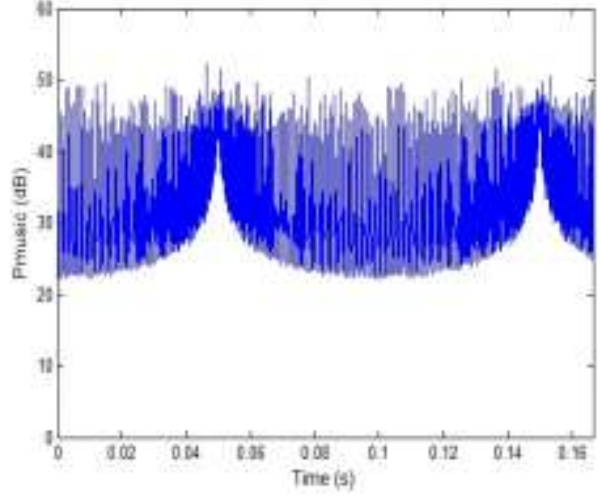

(b)

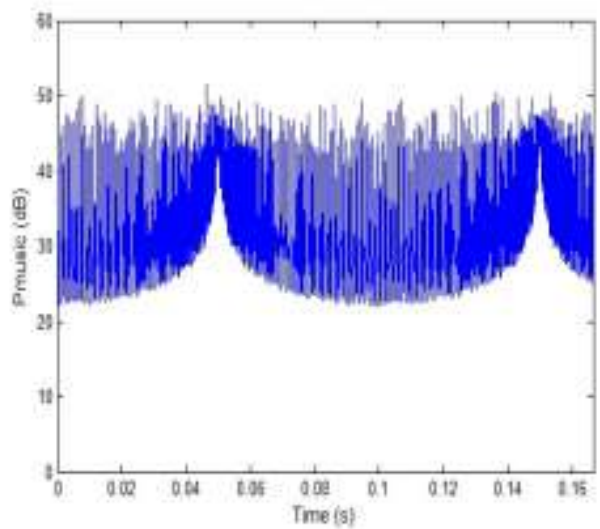

(c)

Figure 7. Spectrum of MUSIC

In Figure 7, (a) (b) (c) show the spectrum of MUSIC as time goes by in three situations: no Doppler Shift, absolute Doppler Shift and relative Doppler Shift. The spectrum height is stable at nearly $50 \mathrm{~dB}$ when there is no Doppler Shift. However different Doppler Shift in channels can make the spectrum fluctuate in a large range $(22 \sim 50 \mathrm{~dB})$, and they also show the periodicity.

Figure 8 shows the phase difference (after MF) between channel 1 and the reference channel (the other channels own similar character). And the period of phase difference coincides with the Doppler Shift.

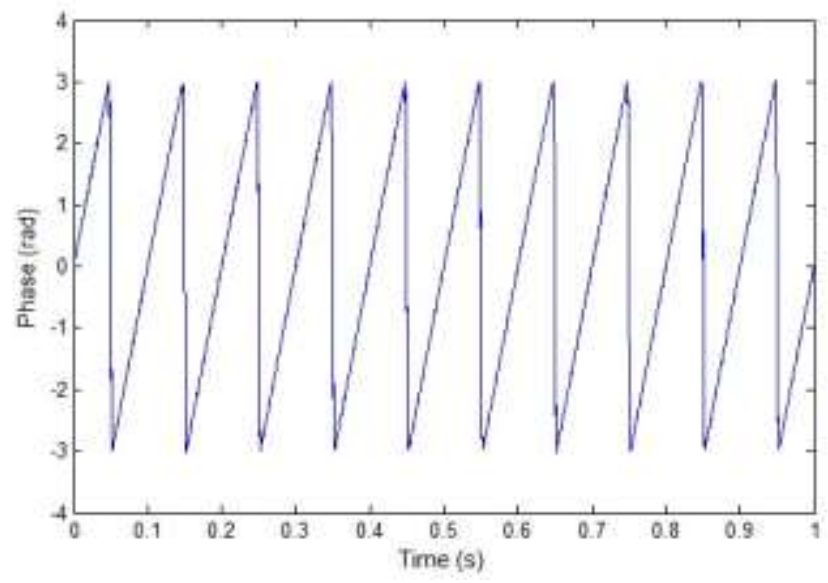

Figure 8. Phase Difference between Channel 1 and Reference Channel 


\subsubsection{Simulation of Compensation Method}

In part 3, improved PLL which is based on EKF is introduced. This structure is added to every channel except for reference channel (channel 0 is set as reference channel).

Figure 9 shows the output $\left(f_{v 1}\right)$ of EKF. Compared with Table 3, the estimation of frequency is almost accurate. And the phase error (channel 1 and 0 ) is also acceptable.
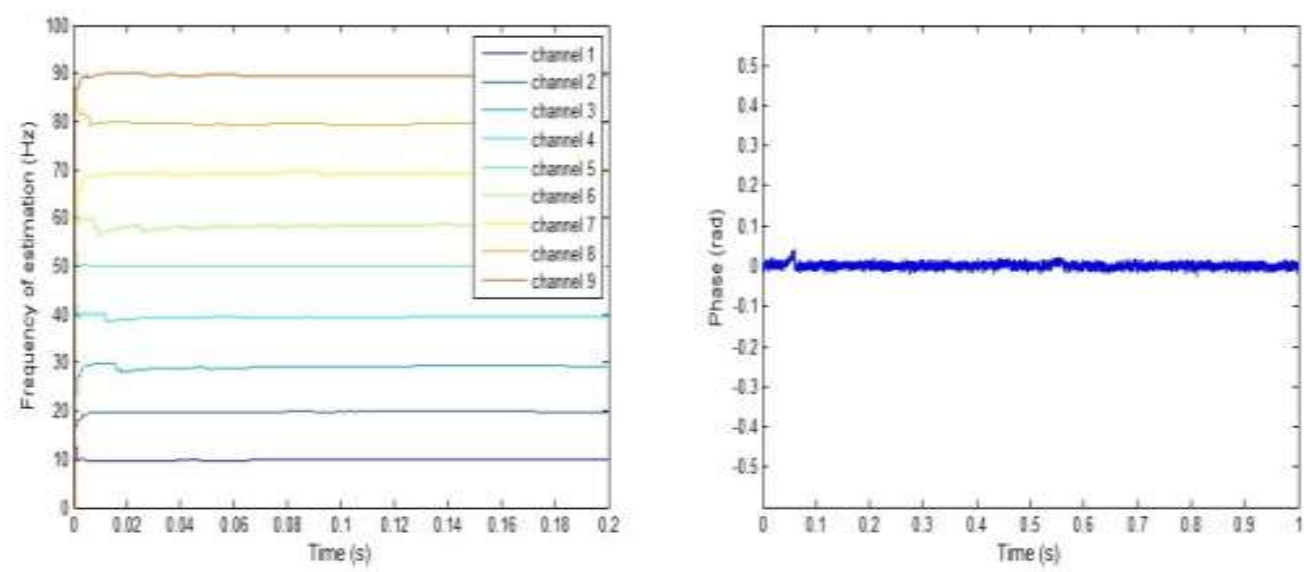

Figure 9. Estimation Frequency and Phase Error

Figure 10 and Figure 11 show the performance of DOA estimation. Compared with Figure $4,5,6$, the direction of MUSIC is stable at $0^{\circ}$ after the compensation. The spectrum of MUSIC is higher and much more stable than before in Figure 7 (b) (c). Because of the noise in channels and the frequency and phase error of the EKF, in Figure 11 , the height of MUSIC spectrum is around $40 \mathrm{~dB}$, which is $10 \mathrm{~dB}$ lower than that in Figure 7 (a).
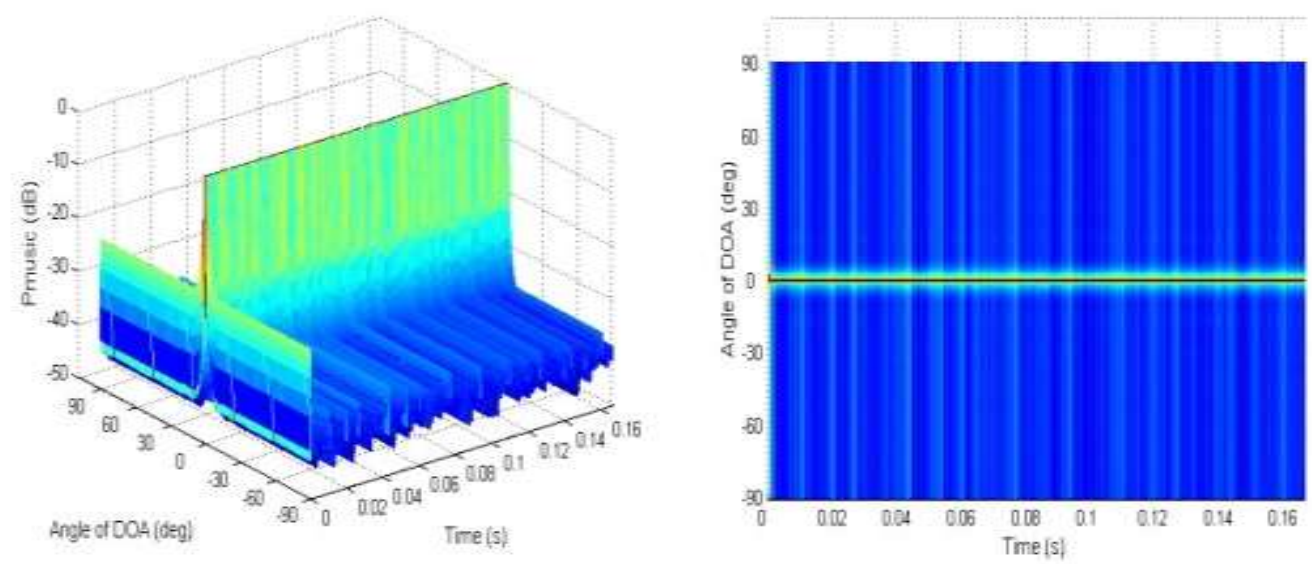

Figure 10. DOA Estimation after Compensation 


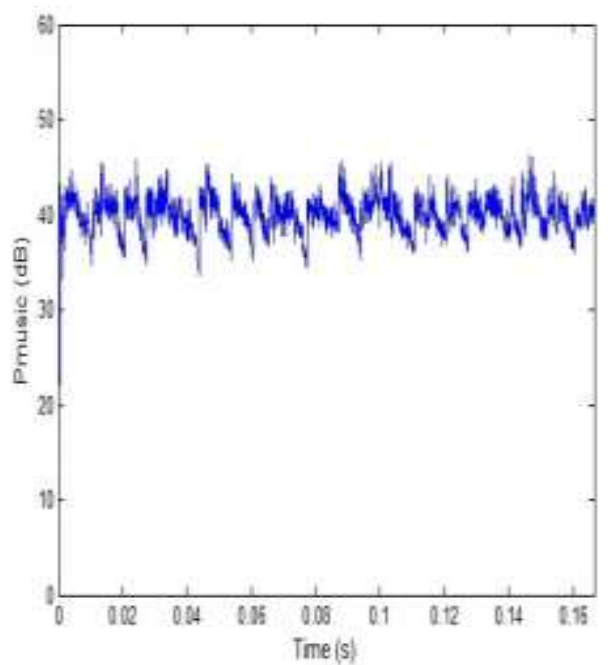

Figure 11. Spectrum of MUSIC after Compensation

\section{Conclusions}

In TDRSS, the FDM method is used to transfer element signals which are received on satellite to the ground station. Relative motion between satellite and ground station can bring different Doppler Shift to 30 channels. As MUSIC algorithm is sensitive to array signals phase error, Doppler Shift in channels can bring great influence on performance of DOA. Through mathematical derivation and simulation, different Doppler Shift in channels makes the estimating direction change periodically. What's more, absolute Doppler Shift and relative Doppler Shift can bring the same effects to DOA estimation and spectral peak. On the basis of that, an improved PLL based on EKF is proposed to compensate different Doppler Shift. By adding the improved PLL structure to every channel except for the reference channel, the relative Doppler Shift among channels can be compensated. Thus it can eliminate the periodical changes in DOA estimation and increase the height of spectrum peak. The theoretical deduction is verified to be correct and the compensation method is effective through computer simulation. This work researches on the problem exists in TDRSS, and may be helpful in designing next generation TDRSS. In the following study, we may improve the compensating accuracy by combining direction error of MUSIC and despreading.

\section{Acknowledgements}

This work was supported by the National Natural Science Foundation of China (No. 61571069), the open research fund of Chongqing key Laboratory of Emergency Communications and Chongqing University Postgraduates' Innovation Project (NO.CYB15030).

\section{References}

[1] P. Angeletti, N. Alagha and S.D. Addio, "Space/ground beamforming techniques for satellite communications", in IEEE APSURSI 2010: Antennas and Propagation Society International Symposium, (2010), pp.1-4.

[2] F.D. Cecca, G. Gallinaro, E. Tirro, C. Campa, S. Cioni and P. Angeletti, "Payload aspects of mobile satellite systems with on-ground beamforming and interference cancellation", in IEEE ICWITS 2012: Wireless Information Technology and Systems, (2012), pp.1-4.

[3] K.L. Du and M.N. Swamy, "Wireless communication systems", Cambridge University Press, Cambridge, UK, (2010). 
[4] D. Roddy, "Satellite communications", Eurasip Journal on Wireless Communications \& Networking, vol. 1, no. 2, (2007), pp.211-216.

[5] S. Amiri and M. Mehdipour, "Accurate Doppler frequency shift estimation for any satellite orbit", in IEEE RAST'07: Recent Advances in Space Technologies, 2007, 3rd International Conference on. IEEE, (2007), pp. 602-607.

[6] S. Liu, H. Li and B. Gou, "DOA estimation error and resolution loss in linear sensor array", in IEEE CISS: Information Sciences and Systems, 2013 47th Annual Conference on. IEEE, (2013), pp. 1-4.

[7] Hakam, R.M. Shubair and E. Salahat, "Enhanced DOA estimation algorithms using MVDR and MUSIC", in IEEE CTIT: Current Trends in Information Technology, 2013 International Conference on. IEEE, (2013), pp.172-176.

[8] H. Paaso, A. Mammela, D. Patron, Damiano, Dandekar and R. Kapil, "DOA estimation through modified unitary MUSIC algorithm for CRLH leaky-wave antennas", in IEEE PIMRC: Personal Indoor and Mobile Radio Communications, 2013 IEEE 24th International Symposium on. IEEE, (2013), pp.311-315.

[9] K. Maeda, T. Nishimura, Y. Ogawa and T. Ohgane, "Accuracy evaluation of DOA estimation with multi-band signals", in IEEE APSURSI: Antennas and Propagation, 2011 IEEE International Symposium on. IEEE, (2011), pp. 835-838.

[10] R.F. Zhang, Y. Liu, X.H. Tan, "Simulation and analysis of the influence of multi-channel group delay distortion on the performance of TDRSS", in Journal of Chongqing University of Posts and Telecommunications (Natural Science), (2011).

[11] X.H. Tan, J. Yu and Y. Liang, "Analysis of effect of AM/PM conversion on DOA estimation performance in TDRSS", in Journal of Computational Information Systems, (2012).

[12] J. Wang, Q. Liang, K. Liang and W. Shangguan, "A new extended Kalman filter based carrier tracking loop", in Microwave, Antenna, Propagation and EMC Technologies for Wireless Communications, 2009 3rd IEEE International Symposium on IEEE, (2009), pp.1181-1184.

[13] W.T. Lin and D.C. Chang, "The extended Kalman filtering algorithm for carrier synchronization and the implementation", in IEEE ISCAS: Circuits and Systems, IEEE International Symposium onIEEE, (2006).

[14] W. Zhang, G.X. Zhang, R.R. Yin, J. Hu and L. Wang, "Doppler Frequency-shift Based on Ephemeris of Satellite", Journal of Military Communications Technology, (2011).

[15] http://celestrak.com/

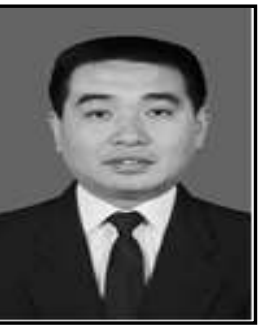

Xiao-Heng Tan, he was born in Chongqing City, China, in 1976. He received the B.S. degree in 1998 and Ph. D. degree in 2003, both from the University of Chongqing, Chongqing, China. He went to the University of Queensland as a visiting scholar during June 2008 to May 2009. He is now a professor and doctoral tutor in the college of communication engineering of Chongqing University, Chongqing, China. His research interests include modern communication technologies and systems (the next generation broad band and wireless mobile communication technology, etc.), communication signal processing.

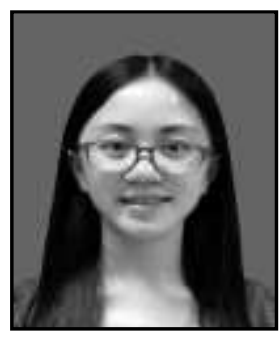

Qian Qu, she was born in Guangan City, China. She received B.S.degree in commnication engineering from Sichuan Normal University, Chengdu, China, in 2016. She is currently studying toward the M.S.degree in Chongqing University, Chongqing, China. Her research interest includes satellite communication. 


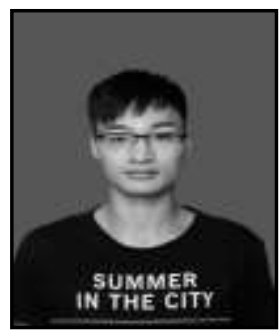

Dong Jiang, he was born in Chongqing City, China. He received his Bachelor degree in electronic information engineering in 2015 and is currently working toward his Master's degree in communication and information system both in Chongqing University, China. $\mathrm{He}$ is mainly researching on communication system, satellite communication.

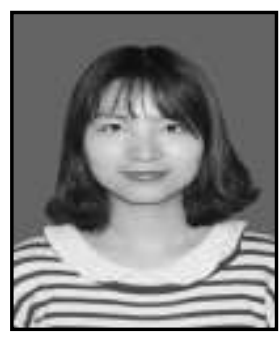

Li Wen, she was born in Hunan province, China. She had been working on circuit test for one year since she received B.S. degree in electronic information engineering in 2014. She is studying for M.S. degree of communication engineering in Chongqing University. Her main research field is satellite communication channel modelling.

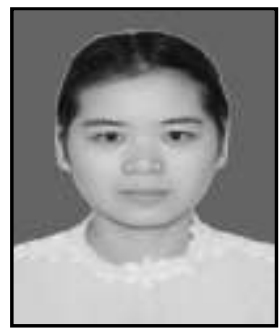

Lu Deng, she was born in Hunan Province, China. She received her Bachelor degree in communication engineering form Dalian University, Dalian, China, in 2016. She is currently working toward her Master's degree in the Field of Electronics and Communication Engineering in Chongqing University, Chongqing, China. She is mainly researching on Computer vision and pattern recognition. 
International Journal of Signal Processing, Image Processing and Pattern Recognition Vol. 10, No. 12 (2017) 